\title{
Intraductal Radiofrequency Ablation for Residual Adenoma after Endoscopic Papillectomy: An Additional Treatment Modality Expected to Be Safe and Effective
}

\author{
Jae Hee Cho \\ Division of Gastroenterology, Department of Internal Medicine, Gangnam Severance Hospital, Yonsei University College of Medicine, \\ Seoul, Korea
}

Corresponding Author

Jae Hee Cho

ORCID https://orcid.org/0000-0003-4174-0091

E-mail jhcho9328@yuhs.ac
See "The Safety of Radiofrequency Ablation Using a Novel Temperature-Controlled Probe for the Treatment of Residual Intraductal Lesions after Endoscopic Papillectomy" by Young Hoon Choi, et al. on page 307, Vol. 15, No. 2, 2021
Ampullary adenoma is rare, but it is increasingly being recognized due to the widespread use of endoscopy and cross-sectional imaging. It is a premalignant neoplasm that is managed by resection. Traditionally, it is managed by surgical resection by either pancreaticoduodenectomy or transduodenal ampullectomy, both of which are associated with significant morbidity and mortality. For these reasons, endoscopic papillectomy (EP) has been explored as an alternative to surgical resection because it avoids surgical morbidity and is relatively safe (complication rate of $18.2 \%$, mortality rate $<0.5 \%) ;{ }^{1}$ however, the blind spot of EP is the intraductal extension of the adenoma into either the bile duct or the pancreatic duct.

Endoscopic retrograde cholangiopancreatography (ERCP)-guided intraductal radiofrequency ablation (IDRFA) has been proposed as an attractive and effective endobiliary ablative therapy because of its ease of delivery, controlled application of thermal energy, low cost, and acceptable safety. ${ }^{2,3}$ In this issue of Gut and Liver, Choi et al. ${ }^{4}$ assessed the efficacy and safety of ERCP-guided IDRFA for the treatment of residual intraductal lesions of ampullary adenoma, which is a critical limitation of EP. They used a $7 \mathrm{~F}$ temperature-controlled catheter (ELRA; STARmed, Goyang, Korea) with a dedicated generator. A clinical ID-RFA pilot study was performed on 10 patients with residual intraductal adenoma (eight patients with low-grade dysplasia and two patients with high-grade dysplasia). The median diameter of the intraductal adenomas was $9 \mathrm{~mm}$ (range, $5-10 \mathrm{~mm}$ ) in the common bile duct
(CBD) and $5 \mathrm{~mm}$ (range, 4-11 $\mathrm{mm}$ ) in the pancreatic duct. The technical success rate was $100 \%$, and no severe adverse events were reported. Only two patients (20\%) had mild post-procedure pancreatitis, and one (10\%) had an asymptomatic biliary stricture. Over a median follow-up period of 253 days, nine patients showed no residual or recurrent adenomatous lesions after ID-RFA.

To date, the therapeutic efficacy of ERCP-guided IDRFA has been evaluated for unresectable malignant biliary obstruction, ${ }^{2,3}$ bridge to surgery, ${ }^{5}$ and residual adenoma in the CBD after EP. ${ }^{6,7}$ To reduce the complications of the conventional ID-RFA systems (Habib, Boston Scientific, Marlborough, MA, USA), a temperature-controlled radiofrequency (RF) catheter (ELRA) has been developed. It automatically adjusts the temperature of the RF catheter tip so that it does not exceed a preset temperature to prevent tissue overheating. This ELRA RFA system was used to confirm safety and usefulness of ID-RFA through preclinical animal experiments and clinical studies. ${ }^{3,8,9}$ Although theoretically, ID-RFA is expected to be an effective local treatment for malignant biliary obstruction, there are still many controversies concerning its benefits in terms of stent patency and survival. The effectiveness of RFA has been demonstrated in a recent large-scale randomized controlled trial. Gao et al. ${ }^{10}$ compared the efficacy and safety between the RFA+ stent group and the stent-only group in patients with unresectable malignant biliary obstruction, such as those with cholangiocarcinoma and ampullary cancer; 174 participants were randomly assigned in 
a 1:1 ratio and completed the two scheduled ERCPs with an interval of approximately 3 months. The median overall survival was significantly higher in the RFA group (14.3 months vs 9.2 months; hazard ratio [HR], 0.49 ; $95 \%$ confidence interval $[\mathrm{CI}], 0.35$ to $0.68 ; \mathrm{p}<0.001$ ). In the subgroup analysis of cholangiocarcinoma, survival benefit was also demonstrated (13.3 months vs 9.2 months; HR, 0.55 ; $95 \%$ CI, 0.39 to $0.77 ; \mathrm{p}<0.001)$. As such, recent research results emphasize the positive therapeutic effect of RFA.

Notably, this study provides the potential of using RFA for the eradication of residual adenoma after EP. Although it is subject to the limitations of retrospective and smallsized endoscopic research, we could find several other interesting discussion points on ID-RFA. First, to the best of our knowledge, this study is the first to investigate temperature-controlled ID-RFA for intraductal residual adenoma after EP. Although the ID-RFA setting was different from the established ID-RFA setting for malignant biliary obstruction (mid to distal CBD $7-10 \mathrm{~W}, 80^{\circ} \mathrm{C}, 2$ minutes), the authors presented a relatively safe ID-RFA setting for intraductal residual adenoma (median RFA time, 65 second; range, 45 to 90 seconds for CBD). In particular, the Habib RF catheter has a long RF ablation zone of approximately $25 \mathrm{~mm}$ in length, which may lead to higher rates of stricture development and an increased risk of severe adverse events. On the other hand, the ELRA 7 W $11 \mathrm{~mm}$ RFA catheter is short in length and can reduce the occurrence of severe adverse events and biliary strictures because it can minimize damage to the normal duct. Second, the safety of ID-RFA within or at the pancreatic orifice is of great concern, particularly in the absence of exophytic disease. But this study suggests a relatively safe ID-RFA setting ( $7 \mathrm{~W}$ : median RFA time, 30 seconds; range, 45 to 90 seconds for pancreatic duct $[\mathrm{PD}])$ for eradication of pancreatic ductal adenomatous extension. Since there are few reports on pancreatic ID-RFA, it might be a safe way that pancreatic ID-RFA followed by the PD stent placement.

This study was retrospective in nature and aimed to evaluate the safety and adjunctive therapeutic role of intraductal RFA after incomplete EP. Given that the alternative to endoscopic management, that is pancreaticoduodenectomy, is not satisfactory, the opportunity to provide patients with a novel endoscopic alternative to ID-RFA is very attractive. In conclusion, endobiliary ID-RFA is a promising treatment option for residual/recurrent intraductal adenoma after EP. Additional controlled studies will be helpful in determining the optimal RFA dosimetry (power, target temperature and duration), clinical benefits, medico-economic validity of ID-RFA, and follow-up strategies after EP.

\section{CONFLICTS OF INTEREST}

No potential conflict of interest relevant to this article was reported.

\section{ORCID}

Jae Hee Cho https://orcid.org/0000-0003-4174-0091

\section{REFERENCES}

1. ASGE Standards of Practice Committee, Chathadi KV, Khashab MA, et al. The role of endoscopy in ampullary and duodenal adenomas. Gastrointest Endosc 2015;82:773-781.

2. Kim EJ, Cho JH, Kim YJ, et al. Intraductal temperaturecontrolled radiofrequency ablation in malignant hilar obstruction: a preliminary study in animals and initial human experience. Endosc Int Open 2019;7:E1293-E1300.

3. Lee YN, Jeong S, Choi HJ, et al. The safety of newly developed automatic temperature-controlled endobiliary radiofrequency ablation system for malignant biliary strictures: a prospective multicenter study. J Gastroenterol Hepatol 2019;34:1454-1459.

4. Choi YH, Yoon SB, Chang JH, Lee IS. The safety of radiofrequency ablation using a novel temperature-controlled probe for the treatment of residual intraductal lesions after endoscopic papillectomy. Gut Liver 2021;15:307-314.

5. Kim EJ, Chung DH, Kim YJ, et al. Endobiliary radiofrequency ablation for distal extrahepatic cholangiocarcinoma: a clinicopathological study. PLoS One 2018;13:e0206694.

6. Rustagi T, Irani S, Reddy DN, et al. Radiofrequency ablation for intraductal extension of ampullary neoplasms. Gastrointest Endosc 2017;86:170-176.

7. Camus M, Napoléon B, Vienne A, et al. Efficacy and safety of endobiliary radiofrequency ablation for the eradication of residual neoplasia after endoscopic papillectomy: a multicenter prospective study. Gastrointest Endosc 2018;88:511-518.

8. Cho JH, Jeong S, Kim EJ, Kim JM, Kim YS, Lee DH. Longterm results of temperature-controlled endobiliary radiofrequency ablation in a normal swine model. Gastrointest Endosc 2018;87:1147-1150.

9. Cho JH, Lee KH, Kim JM, Kim YS, Lee DH, Jeong S. Safety and effectiveness of endobiliary radiofrequency ablation according to the different power and target temperature in a swine model. J Gastroenterol Hepatol 2017;32:521-526.

10. Gao DJ, Yang JF, Ma SR, et al. Endoscopic radiofrequency ablation plus plastic stent placement versus stent placement alone for unresectable extrahepatic biliary cancer: a multicenter randomized controlled trial. Gastrointest Endosc. Epub 2020 Dec 23. http://doi.org/10.1016/j.gie.2020.12.016. 